\title{
Alterations in Esophageal MicroRNAs Expression in Primary Esophageal Achalasia by Next-Generation Sequencing
}

Mahin Gholipour

Golestan University of Medical Sciences and Health Services https://orcid.org/0000-0003-1551-9254

Javad Mikaeli

Tehran University of Medical Sciences

Seyed Javad Mowla

Tarbiat Modares University Faculty of Basic Sciences

Mohammad Reza Bakhtiarizadeh

University of Tehran

Marie Saghaeian Jazi

Golestan University of Medical Sciences and Health Services

Naeme Javid

Golestan University of Medical Sciences and Health Services

Narges Fazlollahi

Tehran University of Medical Sciences

Masoud Khoshnia

Golestan University of Medical Sciences and Health Services Naser Behnampour

Golestan University of Medical Sciences and Health Services

Abdolvahab Moradi ( $\sim$ abmoradi@gmail.com )

Research article

Keywords: hsa-miR-217, Bioinformatics, Gene Expression Analysis, Nerve Degeneration, Achalasia

Posted Date: May 4th, 2020

DOI: https://doi.org/10.21203/rs.2.24777/v2

License: (1) This work is licensed under a Creative Commons Attribution 4.0 International License. Read Full License 


\section{Abstract}

Background: Molecular knowledge regarding the primary esophageal achalasia is essential for early diagnosis and treatment of this neurodegenerative motility disorder. So, there is a need to find the main microRNAs (miRNAs) contributing to the mechanisms of achalasia.

Methods: This study was conducted to determine some patterns of deregulated miRNAs in the achalasia. This case-control study was performed on 52 patients with achalasia and 50 non-achalasia controls. The miRNA expression profiling was conducted on the esophageal tissue samples obtained from the patients with achalasia and non-achalasia patients controls using the Next-Generation Sequencing (NGS). Differential expression of miRNAs was analyzed by the edgeR software. The selected dysregulated miRNAs were additionally confirmed using the quantitative Reverse Transcription Polymerase Chain Reaction (qRT-PCR). Potential target genes of the downregulated and upregulated miRNAs were also predicted to understand the putative role of the miRNAs in the development of the achalasia.

Results: Totally, 15 miRNAs were identified that were significantly altered in the tissues of the patients with achalasia compared to the controls. Among them, three miRNAs including miR-133a-5p, miR-143-3p, and miR-6507-5p were upregulated. Also, six miRNAs including miR-215-5p, miR-216a-5p, miR-216b-5p, miR-217, miR-7641, and miR-194-5p were downregulated significantly. The predicted targets for the dysregulated miRNAs showed significant diseaseassociated pathways like neuronal cell apoptosis, neuromuscular balance, nerve growth factor signaling, and immune response regulation. Gene expression analysis confirmed significant downregulation of the hsa-miR-217 ( $\mathrm{p}$-value $=0.004$ ) in the Lower Esophageal Sphincter (LES) of the patients with achalasia with significant enrichment in the myelination process ontology. The results of this study provide the first integrated miRNA expression profile in the achalasia using the NGS. Our findings introduced 15 candidate miRNAs as achalasia-associated non-coding RNAs and also confirmed the downregulation of the hsamiR-217 in the achalasia disease.

Conclusions: Our results may serve as a basis for more future functional studies to investigate the role of candidate miRNAs in the etiology of achalasia and their application in diagnosis and probably treatment of the disease.

\section{Background}

Achalasia is a chronic neurogenic esophageal motility disorder featured by impaired Lower Esophageal Sphincter (LES) laxity and disturbed peristalsis [1]. Its symptoms include progressive swallowing disorder, regurgitation, esophageal chest pain, aspiration, and eventually malnutrition [2]. According to a population-based study, achalasia prevalence is more than 10/100 000, with a steady increasing trend from 2.51/100 000 in 1996 to $10.82 / 100000$ in 2007. Survival of the patients with achalasia is significantly less than age-sex matched healthy people [2]. Most patients underwent late diagnosis and ineffective treatment due to non-specific symptoms of the disease and the absence of non-invasive diagnostic tests [3].

The pathophysiology of achalasia is based on selective loss of inhibitory neurons in the myenteric network, which can interfere with the coordination of esophageal peristalsis and LES relaxation during swallowing [4]. Decreased levels of the Nitric Oxide Synthase (NOS) and Vasoactive Intestinal Polypeptide (VIP) as inhibitory neurotransmitters in the myenteric plexus disrupt esophageal neuromuscular function in the patients with achalasia [5]. Although the exact mechanism of the disease is not fully understood, some studies have shown the evidence regarding the association of the viral, autoimmune, and neurodegenerative factors [6].

MicroRNAs (miRNA) are a group of small noncoding RNAs acting as gene expression regulators in disease pathways [7]. The miRNA system is involved in various physiological and pathophysiological processes, and miRNAs are used as potential prognostic biomarkers [8]. Several studies have shown alterations in the expression of miRNAs in various disorders, including cancers [9], immune-mediated inflammatory diseases [10], and nervous disturbances [11].

It is well known that the achalasia pathogenesis is associated with neurological communication, cholinergic signaling, and inflammation. On the other hand, miRNAs expression influences all of these processes [12]. Thus, studying miRNAs expression could help us better understand the achalasia pathophysiology. Although investigating the effects of miRNAs on the pathogenesis of several diseases has received considerable attention, their effects on the development of the achalasia are still unclear. So, in the present study, the Next-Generation Sequencing (NGS) was performed with an analytical approach to identify reliable candidate miRNAs associated with the development of the disease.

\section{Methods}

\section{Participants and Sampling}

This matched case-control study was performed on 102 patients referred to the Digestive Diseases Research Center (DDRC) in the Shariati Hospital in TehranIran between August 2015 and April 2016. All the patients with primary esophageal achalasia ( $\mathrm{N}=52$ cases diagnosed based on the clinical, radiological, endoscopic findings and high-resolution manometry, and aged $\geq 18$ years old) who referred to the clinic for regular follow-up were consecutively recruited in this study. All the patients received the same pneumatic dilatation treatment to decrease the confounding factors and then, the patients were classified into excellent, good, moderate, and poor categories according to the outcome. Patients in the excellent and good categories were considered as the patients with good response to the treatment, and the patients in the moderate and poor categories were considered as those with poor response to the treatment [13]. Controls $(\mathrm{N}=50)$ were selected randomly from the individuals without dysphagia or lesions in the esophagus visiting the same clinic. All the cases and controls were matched by age ( \pm 5 years) and sex. Participants underwent the endoscopy and biopsy samples were prepared from the LES by an expert clinician. The samples were stored at $-80^{\circ} \mathrm{C}$ for subsequent experiments. Patients with other associated motility or non-motility disorders, malignancy, and coagulopathy were excluded from the study.

\section{RNA Isolation and Deep Sequencing}


Total RNA was extracted from all the samples (52 cases and 50 controls) using the Trizol reagent according to the manufacturers' instructions (Invitrogen, Sweden). RNA samples obtained from each group of participants including those with excellent, good, moderate, and poor responses to the treatment were pooled (mixed equally) and were sent for miRNA sequencing to increase the experiment power. For the controls, the RNA samples were also pooled equally in two samples and then, were sent for miRNA profiling.

The samples were sent to the BGI (Beijing Genomics Institute), a Chinese genome sequencing company for miRNA sequencing. Also, Bioanalyzer 2100 (Agilent, Santa Clara, CA) was employed to measure the RNA Integrity Number (RIN) for each sample and the samples with RIN greater than seven were considered for sequencing. RNA purification, library construction, and sequencing were also conducted by the BGI Company. Each library was single-end sequenced on an Illumina HiSeq 4000 platform. The raw miRNA-Seq data were deposited and released in the Sequence Read Archive (SRA) database, with the BioProject accession number of PRJNA616451.

\section{Analysis of Small RNA Sequencing Data (NGS Data)}

FASTQC (version 0.11.5; http://www.bioinformatics.babraham.ac.uk/projects/fastqc/) was used to perform primary quality control of the miRNA-Seq data. Afterward, low-quality reads and adapter sequences of raw data were trimmed by the Trimmomatic software (version 0.35) [14] (parameters of trailing 20, maxinfo 18:0.90, and minimum length 18). Reads with the length shorter than 18 bases were discarded after quality trimming, and the remaining reads were mapped against the Rfam database (http://rfam.xfam.org/) to eliminate unwanted noncoding RNAs ( rRNAs, tRNAs, snRNAs, and snoRNA). Subsequently, the remaining reads were analyzed using the miRDeep2 software (version 0.0.8)[15] to quantify known miRNAs and predict novel miRNAs. For efficient read mapping, clean reads in each sample were collapsed into a set of unique sequences with read numbers counted. Then, the unique sequences were aligned to the Ensembl GRCH37 human genome (http://grch37.ensembl.org) and miRNAs sequences (miRBase database, version 21) [16]. The aligned reads were quantified using the default settings of the miRDeep2 software that was allowed only one mismatch within the read. On the other hand, putative novel miRNAs were predicted using the default settings in the miRDeep2 software. The predictions by the miRDeep2 software were filtered, with a miRDeep2 score of $>1$, the length $\geq 50$ nucleotides, and the predicted probability of being a miRNA $>60 \%$. The difference in miRNAs expression (fold change) was analyzed by the edgeR package (v1.4.5) in the R software. Fold change with adjusted p-value or False Discovery Rate (FDR) of less than 0.05 was considered as statistically significant.

\section{MiRNAs Target Prediction and Gene Enrichment Analyses}

Potential target genes of the differentially expressed miRNAs were predicted using the three target prediction programs including PITA[17], TargetSpy [18], and RNAhybrid [19]. If a gene was predicted by at least two used programs, it was considered as a putative target. Since every software has different levels of sensitivity and specificity, using the combination of these programs reduced the false positive. Every software uses various algorithms to predict miRNA targets. The default parameters of the software were applied. The 3'UTR sequences were recovered by the Ensembl BioMart (http://www.ensembl.org/Multi/martview) and then, were used for prediction. Finally, Gene Ontology (GO) and Kyoto Encyclopedia Genes and Genomes (KEGG, http://www.genome.ad.jp/kegg) were applied to analyze the potential function and pathway of target genes.

\section{quantitative Reverse Transcription Polymerase Chain Reaction (qRT-PCR) Analysis}

For more confirmation, the expression level of the dysregulated miRNAs was measured in the esophagus tissues of the patients with achalasia and control individuals by the ABI 7300 real-time PCR machine (Applied Biosystems, USA). cDNA synthesis of miRNAs was performed by the Reverse Transcription System Kit (Zist Royesh, Iran) with a miR-specific stem-loop primer [20]. The SNORD47 was measured as an internal normalization control using the 2-dct method. For qRT-PCR statistical analysis, differences between the two groups were tested by Student's t-test and the Mann-Whitney U test (based on normality of data distribution) in the SPSS statistical software version 16.0. The difference with a probability value of less than 0.05 was considered as statistically significant.

\section{Results}

\section{Esophageal Tissues of the Patients with Achalasia Expressed miRNA Profile Different from the Controls}

Table 1 summarizes the clinical information of the patients. As demonstrated in Table 1 , there is no significant difference in the age $(p-v a l u e=0.48)$ and sex ( $p$-value $=0.43$ ) between the cases and controls. The miRNA sequencing results were compared between three groups: good response group including pooled samples 1 and 2 (those with good and excellent response to the dilatation treatment), poor response group consisting of pooled samples 3 and 4 (those with moderate and poor response to the dilatation treatment), and pooled samples 5 and 6 that were merged into control group to perform the transcriptome analysis based on the clinicians' recommendation (Table 2). It was attempted to increase the statistical power through post-processing replication for each group.

Table 1 Clinical data for 44 achalasia patients and 45 controls 


\begin{tabular}{|c|c|c|c|}
\hline Characteristic & Patients $\dagger$ & Controls $\dagger$ & p-value \\
\hline Mean Age (SD ), year & $43.5(1.6)$ & $45.8(1.6)$ & 0.48 \\
\hline Male/Female No. (\% male) & $31 / 21(59.6)$ & $26 / 24(52)$ & 0.43 \\
\hline Vantrappen Classification§ & n (\%) & & \\
\hline Excellent & $15(28.8)$ & & \\
\hline Good & $15(28.8)$ & & \\
\hline Moderate & $12(23.1)$ & & \\
\hline Poor & $10(19.2)$ & & \\
\hline Achalasia subtype & n (\%) & & \\
\hline Type 1 & $9(17.3)$ & & \\
\hline Type 2 & $42(80.8)$ & & \\
\hline Type 3 & $1(1.9)$ & & \\
\hline Mean duration (months) of symptoms (SD) & $32.34(2.06)$ & & \\
\hline Baseline symptoms & n (\%) & & \\
\hline Dysphagia & $43(82.7)$ & & \\
\hline Chest pain & $7(13.5)$ & & \\
\hline Regurgitation & $2(3.8)$ & & \\
\hline
\end{tabular}

† Unless otherwise indicated data are expressed as number (percentage) of patients. Percentages have been rounded and might not total 100. ¥ SD: Standard Deviation

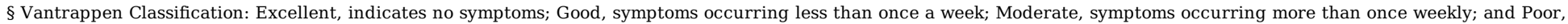
persistent symptoms [21]

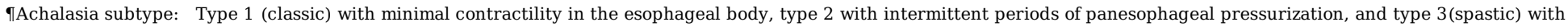
premature or spastic distal esophageal contractions[22]

Table 2 Next-Generation Sequencing read counts and Mapping Result for individual samples

\begin{tabular}{|c|l|l|l|l|l|l|}
\hline Post-processing grouping & Clinical outcome after dilatation & Sample ID & Total Reads & Mapped Reads & Mapped (\%) \\
\hline \multirow{2}{*}{1} & Good response to treatment & Excellent & Pooled Sample_1 & 30378288 & 15879232 \\
\cline { 3 - 6 } & & Good & Pooled Sample_2 & 26809904 & 13712314 \\
\hline 2 & Poor response to treatment & Moderate & Pooled Sample_3 & 29249887 & 14814455 \\
\cline { 3 - 6 } & & Poor & Pooled Sample_4 & 30445887 & 16872768 & 0.511 \\
\hline \multirow{2}{*}{3} & Without treatment / Control & Control 1 & Pooled Sample_5 & 24835372 & 10948105 & 0.554 \\
\cline { 3 - 6 } & & Control 2 & Pooled Sample_6 & 29473046 & 13101058 & 0.445 \\
\hline
\end{tabular}

The miRNA expression profiling analysis showed that 15 miRNAs were significantly differentially expressed in the tissues of the patients with achalasia (good or poor response groups) compared to the controls. Besides, our findings indicated that most of the dysregulated miRNAs (11 miRNAs) were downregulated and four miRNAs were upregulated in the tissues of the patients with achalasia. Three miRNAs were significantly upregulated in both good and poor response group compared to the controls; miR-133a-5p (adjusted p-value $<0.001$ for good response group \& adjusted $p$-value $=0.005$ for poor response group), miR$143-3 p$ (adjusted p-value $=0.001$ for good response group \& adjusted $p$-value $=0.011$ for poor response group) and miR- $6507-5 p($ adjusted $p$-value $=0.001$ for good response group \& adjusted p-value $=0.016$ for poor response group). Also, the NGS data showed hsa-miR-3609 was significantly upregulated just in a good response group compared to the controls (adjusted p-value $=0.021$ ). Furthermore, we found six miRNAs that were downregulated significantly in both good and poor response groups (Fig.1). These were miR-215-5p, miR-216a-5p, miR-216b-5p, miR-217 and miR-7641 with adjusted p-value < 0.001 and miR194-5p (adjusted p-value $=0.01$ for good response group \& adjusted p-value $=0.005$ for poor response group). However, the good response group showed significant downregulation in the expression of four miRNAs including hsa-miR-135a-5p, hsa-miR-4488, hsa-miR-122-5p, hsa-miR-4449 compared to the controls. On the other hand, the significant downregulation of the hsa-miR-383-5p (adjusted p-value $=0.001$ ) was seen in poor response achalasia group compared to the controls (Table 3). These findings highlight the potential prognostic value of the candidate miRNAs for treatment outcome prediction. This study did not detect significant differential expression in any of the miRNAs between two groups of patients with achalasia (good and poor response groups) regarding the treatment outcome.

Table 3 Fifteen significant upregulated and downregulated miRNAs in the achalasia tissues (Good response group \& Poor response group) versus control tissues

\begin{tabular}{|c|c|c|c|c|c|c|c|c|}
\hline \multirow[t]{2}{*}{ MicroRNA } & \multicolumn{4}{|c|}{ Good response group } & \multicolumn{4}{|c|}{ Poor response group } \\
\hline & $\mathrm{FC}^{\dagger}$ & $\log 2 \mathrm{FC}^{\dagger}$ & p-value & a p-value* & $\mathrm{FC}^{\dagger}$ & $\log 2 \mathrm{FC}^{\dagger}$ & $\mathrm{P}$ value & a p-value* \\
\hline hsa-miR-217 & $\downarrow 0.020$ & -5.644 & $3.98 \mathrm{E}-10$ & $2.46 \mathrm{E}-07$ & $\downarrow 0.31$ & -1.69 & 9.68E-09 & $1.5 \mathrm{E}-06$ \\
\hline hsa-miR-216a-5p & $\downarrow 0.062$ & -4.011 & $1.05 \mathrm{E}-09$ & $2.65 \mathrm{E}-07$ & $\downarrow 0.047$ & -4.411 & $1.02 \mathrm{E}-10$ & $3.14 \mathrm{E}-08$ \\
\hline hsa-miR-7641 & $\downarrow 0.155$ & -2.689 & $1.28 \mathrm{E}-09$ & $2.65 \mathrm{E}-07$ & $\downarrow 0.160$ & -2.644 & $2.28 \mathrm{E}-09$ & $4.71 \mathrm{E}-07$ \\
\hline hsa-miR-216b-5p & $\downarrow 0.08$ & -3.644 & $2.06 \mathrm{E}-09$ & $3.18 \mathrm{E}-07$ & $\downarrow 0.04$ & -4.644 & $7.52 \mathrm{E}-13$ & $4.65 \mathrm{E}-10$ \\
\hline hsa-miR-215-5p & $\downarrow 0.240$ & -2.059 & $8.98 \mathrm{E}-07$ & 0.000111 & $\downarrow 0.193$ & -2.373 & $2.39 \mathrm{E}-08$ & $2.95 \mathrm{E}-06$ \\
\hline hsa-miR-135a-5p & $\downarrow 0.173$ & -2.531 & $2.14 \mathrm{E}-06$ & 0.00022 & - & - & - & - \\
\hline hsa-miR-194-5p & $\downarrow 0.368$ & -1.442 & 0.000174 & 0.010725 & $\downarrow 0.353$ & -1.502 & 7.62E-05 & 0.005888 \\
\hline hsa-miR-4488 & $\downarrow 0.323$ & -1.630 & 0.000571 & 0.029432 & - & - & - & - \\
\hline hsa-miR-122-5p & $\downarrow 0.231$ & -2.114 & 0.000723 & 0.03438 & - & - & - & - \\
\hline hsa-miR-4449 & $\downarrow 0.302$ & -1.727 & 0.000835 & 0.036864 & - & - & - & - \\
\hline hsa-miR-133a-5p & $\uparrow 35$ & 5.129 & $2.89 \mathrm{E}-06$ & 0.000255 & $\uparrow 19$ & 4.248 & 7.62E-05 & 0.005888 \\
\hline hsa-miR-143-3p & $\uparrow 6.702$ & 2.744 & $1.74 \mathrm{E}-05$ & 0.001345 & $\uparrow 5.173$ & 2.371 & 0.000166 & 0.011374 \\
\hline hsa-miR-6507-5p & $\uparrow 44$ & 5.459 & $2.36 \mathrm{E}-05$ & 0.00162 & $\uparrow 24$ & 4.585 & 0.000261 & 0.016122 \\
\hline hsa-miR-3609 & $\uparrow 4.6$ & 2.202 & 0.00038 & 0.021343 & - & - & - & - \\
\hline hsa-miR-383-5p & - & - & - & - & $\downarrow 0.133$ & -2.910 & $1.28 \mathrm{E}-05$ & 0.001317 \\
\hline
\end{tabular}

${ }^{\dagger} \mathrm{FC}$, Fold change; *a p-value ${ }^{\S}$, adjusted p-value 
The biological process of GO and KEGG pathways of the 15 candidate miRNAs were analyzed based on the biological process. As detailed in Table 4, we introduced a list of the most significantly enriched terms and pathways .of the target genes of candidate miRNAs involved in achalasia. Interestingly, GO analysis showed that the differentially expressed genes associated with the neuron apoptotic process (adjusted p-value $=0.004$ ), neuronal death (adjusted $p$ value $=0.006$ ), and immune response regulation (adjusted $p$-value $=0.008$ ) were targeted by hsa-miR-143-3p. It was also found that the genes related to the cellular response to oxidative stress (adjusted $p$-value $=0.011$ ), cellular aging (adjusted $p$-value $=0.011$ ), axon regeneration and development (adjusted $p$ value $=0.031$ ) and myelination (adjusted $p$-value $=0.031$ ) were significantly enriched by the hsa-miR-217. Moreover, KEGG analysis showed that the genes involved in Glioma (adjusted p-value $=0.0001$ ) and the Sphingolipid signaling pathway (adjusted p-value $=0.0006$ ) were the most highly represented pathways enriched by the hsa-miR-143-3p. Also, the genes associated with cancers including non-small cell lung cancer (adjusted $p$-value $=0.001$ for hsa-miR143-3p \& adjusted p-value $=0.004$ for hsa-miR-217), prostate (adjusted p-value $=0.004$ for hsa-miR-217), colorectal (adjusted p-value $=0.001$ for hsa-miR-143$3 p$ ), bladder (adjusted p-value $=0.001$ for hsa-miR-143-3p) and endometrial cancers (adjusted p-value $=0.004$ for hsa-miR-217) were significantly enriched by the predicted target genes (Table 4).

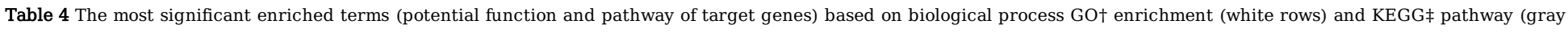
rows) of the miRNAs associated with achalasia 


\begin{tabular}{|c|c|c|}
\hline miRNA & enriched Term & Target genes \\
\hline \multirow{6}{*}{$\begin{array}{l}\text { hsa- } \\
\text { miR- } \\
217\end{array}$} & $\begin{array}{l}\text { Non-small cell lung cancer- Homo sapiens- } \\
\text { hsa05223 }\end{array}$ & E2F3;KRAS;FOX3;FHIT \\
\hline & Endometrial cancer- Homo sapiens- hsa05213 & TCF7L2;PTEN;KRAS;FOXO3 \\
\hline & $\begin{array}{l}\text { Negative regulation of cell aging } \\
\text { (GO:0090344) }\end{array}$ & PTEN; SIRT1; MARCH 5 \\
\hline & $\begin{array}{l}\text { Cellular response to oxidative stress } \\
\text { (GO:0034599) }\end{array}$ & NR4A2;TP53INP1;FOXO3;SIRT1;HIF1A;EZH2 \\
\hline & Prostate cancer- Homo sapiens-hsa05215 & TCF7L2;PTEN;E2F3;KRAS \\
\hline & Regulation of myelination (GO:0031641) & TCF7L2;PTEN;TNFRSF21 \\
\hline \multirow{4}{*}{$\begin{array}{l}\text { hsa- } \\
\text { miR- } \\
216 b- \\
5 p\end{array}$} & Melanoma- Homo sapiens- hsa05218 & CDK6;CDK4;MAPK1;KRAS;FGF10 \\
\hline & Pathways in cancer-Homo sapiens-hsa05200 & CDK6;FZD5;TPM3;CDK4;COL4A4;FZD9;TCEB2;MAPK1;KRAS;FGF10 \\
\hline & $\begin{array}{l}\text { Signaling pathways regulating pluripotency of } \\
\text { stem cells-Homo sapiens_hsa04550 }\end{array}$ & SMAD1;FZD5;FZD9;MAPK1;LHX5;KRAS \\
\hline & $\begin{array}{l}\text { Non-small cell lung cancer-Homo sapiens- } \\
\text { hsa05223 }\end{array}$ & CDK6;CDK4;MAPK1;KRAS \\
\hline $\begin{array}{l}\text { hsa- } \\
\text { miR- } \\
215-5 p\end{array}$ & Cell cycle-Homo sapiens-hsa04110 & RB1;CDKN2D;CDKN2A;BUB1B;CDC7;TTK;CDC14A;ANAPC10;CDC20;ORC4;ORC1;CCNE1;RAD21;MCM3;MCM6;MAI \\
\hline \multirow{10}{*}{$\begin{array}{l}\text { hsa- } \\
\text { miR- } \\
143-3 p\end{array}$} & Glioma-Homo sapiens- hsa05214 & PDGFRA;MDM2;AKT1;MAPK1;BRAF;CALM3;KRAS;HRAS;IGF1R \\
\hline & $\begin{array}{l}\text { Sphingolipid signaling pathway- Homo sapiens- } \\
\text { hsa04071 }\end{array}$ & CERS4;SGPL1;SPTLC2;PPP2R5E;BCL2;AKT1;MAPK1;KRAS;TNF;HRAS \\
\hline & MicroRNAs in cancer- Homo sapiens-has 05206 & TRIM71;PDGFRA;DNMT3A;PTGS2;MAPK7;ERBB3;FSCN1;MDM2;BCL2;MAPK1;KRAS;HRAS;CD44 \\
\hline & $\begin{array}{l}\text { Non-small cell lung cancer-Homo sapiens- } \\
\text { hsa05223 }\end{array}$ & AKT1;MAPK1;BRAF;KRAS;FHIT;HRAS \\
\hline & Colorectal cancer-Homo sapiens-hsa05210 & SMAD3;BCL2;AKT1;MAPK1;BRAF;KRAS \\
\hline & Bladder cancer-Homo sapiens-hsa05219 & MDM2;MAPK1;BRAF;KRAS;HRAS \\
\hline & Regulation of neuron death (GO:1901214) & ERBB3;UBE2V2;BCL2;AKT1;XIAP;KRAS;BRAF;HRAS;TNF;BB \\
\hline & $\begin{array}{l}\text { Regulation of neuron apoptotic process } \\
\text { (GO:0043523) }\end{array}$ & ERBB3;UBE2V2;BCL2;XIAP;KRAS;BRAF;HRAS;TNF;BBC3 \\
\hline & $\begin{array}{l}\text { Negative regulation of neuron death } \\
\text { (GO:1901215) }\end{array}$ & ERBB3;UBE2V2;BCL2;AKT1;XIAP;KRAS;BRAF;HRAS \\
\hline & $\begin{array}{l}\text { Immune response regulating cell surface } \\
\text { receptor signaling pathway GO:0002768) }\end{array}$ & PDGFRA;NCKAP1;PLEKHA1;YWHAB;LIMK1;ERBB \\
\hline $\begin{array}{l}\text { hsa- } \\
\text { miR- } \\
6507- \\
5 p \\
\end{array}$ & cytokinesis_(GO:0000910) & RACGAP1;PRC1;NEK7;MYH9;CEP55;RHOB \\
\hline \multirow{11}{*}{$\begin{array}{l}\text { hsa- } \\
\text { miR- } \\
135 a- \\
5 p\end{array}$} & $\begin{array}{l}\text { Jak-STAT signaling pathway-Homo sapiens- } \\
\text { hsa04630 }\end{array}$ & PIAS4;MYC;MPL;BCL2;STAT6;JAK2 \\
\hline & $\begin{array}{l}\text { Signaling pathways regulating pluripotency of } \\
\text { stem cells-Homo sapiens-hsa04550 }\end{array}$ & BMPR2;APC;MYC;JAK2;SMAD5;SKIL \\
\hline & Colorectal cancer-Homo sapiens-hsa05210 & APC;MYC;BCL2;BIRC5 \\
\hline & MicroRNAs in cancer-Homo sapiens-hsa05206 & MARCKS;BMPR2;APC;ROCK1;MYC;BCL2;IRS2 \\
\hline & $\begin{array}{l}\text { TGF beta signaling pathway-Homo sapiens- } \\
\text { hsa04350 }\end{array}$ & BMPR2;ROCK1;MYC;SMAD5 \\
\hline & $\begin{array}{l}\text { Cellular response to BMP stimulus } \\
\text { (GO:0071773) }\end{array}$ & HEYL;GATA6;SMAD5 \\
\hline & Response to BMP (GO:0071772) & HEYL;GATA6;SMAD5 \\
\hline & neuron_projection_regeneration_(GO:0031102) & BCL2;APOA1;JAK2 \\
\hline & Axon development (GO:0061564) & BCL2;APOA1;JAK2 \\
\hline & Axon regeneration (GO:0031103) & BCL2;APOA1;JAK2 \\
\hline & $\begin{array}{l}\text { Positive regulation of ntrinsic apoptotic } \\
\text { signaling pathway (GO:2001244 }\end{array}$ & PIAS4;SIAH1;BCL2;SKIL \\
\hline \multirow{4}{*}{$\begin{array}{l}\text { hsa- } \\
\text { miR- } \\
3609\end{array}$} & Pathways in cancer-Homo sapiens-hsa05200 & ITGB1;EGLN3;PRKCB;F2R;FZD9;XIAP;HIF1A;IGF1R;TGFBR2;BMP2;CCND1;MDM2;MAPK1;CRK;APPL1;F2RL3 \\
\hline & $\begin{array}{l}\text { Proteoglycans in cancer Homo sapiens } \\
\text { hsa05205 }\end{array}$ & ITGB1;CCND1;PRKCB;CAV1;FZD9;MDM2;RRAS2;MAPK1;HIF1A;THBS1;IGF1R \\
\hline & Endocytosis-Homo sapiens hsa04144 & SH3GLB1;HSPA8;RAB5B;RAB4A;ZFYVE9;SH3KBP1;CAV1;F2R;EPS15L1;IGF1R;TGFBR2;RAB11FIP1;MDM2 \\
\hline & Focal adhesion- Homo sapiens-hsa04510 & RAP1B;ITGB1;CCND1;PRKCB;CAV1;XIAP;PAK6;MAPK1;CRK;THBS1;IGF1R \\
\hline \multirow{4}{*}{$\begin{array}{l}\text { hsa- } \\
\text { miR- } \\
\text { 194-5p }\end{array}$} & Adherens junction- Homo sapiens-hsa04520 & TJP1;EP300;RAC1;IGF1R \\
\hline & $\begin{array}{l}\text { Proteoglycans in cancer-Homo sapiens- } \\
\text { hsa05205 }\end{array}$ & CAV1;FZD6;RAC1;HBEGF;IGF1R \\
\hline & Focal adhesion- Homo sapiens-hsa04510 & CAV1;TLN2;RAC1;IGF1R;ITGA9 \\
\hline & $\begin{array}{l}\text { HIF-1 signaling pathway-Homo sapiens- } \\
\text { hsa04066 }\end{array}$ & CDKN1B;EP300;RBX1;IGF1R \\
\hline
\end{tabular}

${ }^{\dagger}$ GO, Gene Ontology; ${ }^{\ddagger}$ KEGG, Kyoto Encyclopedia of Genes and Genomes; *a p-value, adjusted p-value 


\section{Novel Predicted miRNAs in the Esophageal Tissue}

Interestingly, the data analysis showed novel potential miRNA transcripts in the esophageal tissues, which were expressed in at least two different pooled samples with mean read counts per group greater than five. All the rRNAs and tRNAs were excluded by the Rfam software

(http://www.sanger.ac.uk/Software/Rfam/) and the identified novel miRNAs possessed the criteria of secondary structure in the RNA fold change. Thirty-six novel candidate miRNAs were identified with mammalian homologues using this approach (Table S1), but none of them was significantly changed in the achalasia. GO analysis showed that eight novel miRNAs are significantly related to the neurotransmission process (adjusted p-value 0.03), axon development and regeneration (adjusted p-value 0.02), cellular response to nerve growth factor (adjusted p-value 0.03), and inflammation process (Table 5).

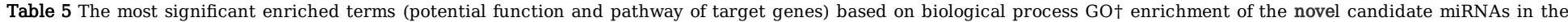
esophageal tissues

\begin{tabular}{|c|c|c|c|}
\hline miRNA & enriched Term & Target genes & a p-value $^{*}$ \\
\hline \multirow[t]{6}{*}{$2: 46348793 . .46348872$} & positive_regulation_of_neurotransmitter_transport_(GO:0051590) & \multirow[t]{6}{*}{ DTNBP1 } & 0.024 \\
\hline & positive_regulation_of_neurotransmitter_secretion_(GO:0001956) & & 0.024 \\
\hline & anterograde_axon_cargo_transport_(GO:0008089) & & 0.024 \\
\hline & axon_cargo_transport_(GO:0008088) & & 0.03 \\
\hline & regulation_of_neurotransmitter_secretion_(GO:0046928) & & 0.03 \\
\hline & regulation_of_neurotransmitter_transport_(GO:0051588) & & 0.03 \\
\hline 3:186787298..186787358 & neuroepithelial_cell_differentiation_(GO:0060563) & MITF & 0.046 \\
\hline \multirow[t]{3}{*}{$6: 104646203 . .104646269$} & cellular_response_to_interleukin-6_(GO:0071354) & \multirow[t]{3}{*}{ GALT } & 0.039 \\
\hline & interleukin-6-mediated_signaling_pathway_(GO:0070102) & & 0.027 \\
\hline & response_to_interleukin-6_(GO:0070741) & & 0.04 \\
\hline \multirow[t]{2}{*}{$7: 53776229 . .53776317$} & positive_regulation_of_interleukin-8_biosynthetic_process_(GO:0045416) & \multirow[t]{2}{*}{ PRG3 } & 0.031 \\
\hline & regulation_of_interleukin-8_production_(GO:0032677) & & 0.031 \\
\hline \multirow[t]{16}{*}{ 15:60128283..60128360 } & neuron_projection_regeneration_(GO:0031102) & \multirow[t]{16}{*}{ NEFL } & 0.02 \\
\hline & axon_development_(GO:0061564) & & 0.02 \\
\hline & axon_regeneration_(GO:0031103) & & 0.02 \\
\hline & anterograde_axon_cargo_transport_(GO:0008089) & & 0.02 \\
\hline & neurofilament_cytoskeleton_organization_(GO:0060052) & & 0.02 \\
\hline & axon_cargo_transport_(GO:0008088) & & 0.02 \\
\hline & response_to_axon_injury_(GO:0048678) & & 0.02 \\
\hline & positive_regulation_of_axonogenesis_(GO:0050772) & & 0.024 \\
\hline & negative_regulation_of_neuron_apoptotic_process_(GO:0043524) & & 0.036 \\
\hline & regulation_of_axonogenesis_(GO:0050770) & & 0.036 \\
\hline & negative_regulation_of_neuron_death_(GO:1901215) & & 0.037 \\
\hline & regulation_of_neuron_apoptotic_process_(GO:0043523) & & 0.04 \\
\hline & positive_regulation_of_neuron_differentiation_(GO:0045666) & & 0.044 \\
\hline & regulation_of_neuron_death_(GO:1901214) & & 0.044 \\
\hline & regulation_of_neuron_projection_development_(GO:0010975) & & 0.046 \\
\hline & positive_regulation_of_neurogenesis_(GO:0050769) & & 0.047 \\
\hline \multirow[t]{7}{*}{$20: 38425194 . .38425268$} & cellular_response_to_nerve_growth_factor_stimulus_(GO:1990090) & RAP1A & 0.032 \\
\hline & response_to_nerve_growth_factor_(GO:1990089) & RAP1A & 0.032 \\
\hline & positive_regulation_of_calcium_ion_transmembrane_transporter_activity_(GO:1901021) & ANK2 & 0.032 \\
\hline & negative_regulation_of_neurotransmitter_transport_(GO:0051589) & RAP1A & 0.032 \\
\hline & nerve_growth_factor_signaling_pathway_(GO:0038180) & RAP1A & 0.032 \\
\hline & negative_regulation_of_neurotransmitter_secretion_(GO:0046929) & RAP1A & 0.032 \\
\hline & regulation_of_neurotransmitter_secretion_(GO:0046928) & RAP1A & 0.047 \\
\hline \multirow[t]{6}{*}{$6: 77781479 . .77781527$} & regulation_of_intrinsic_apoptotic_signaling_pathway_by_p53_class_mediator_(GO:1902253) & \multirow[t]{6}{*}{ RRM2B } & 0.004 \\
\hline & negative_regulation_of_signal_transduction_by_p53_class_mediator_(GO:1901797) & & 0.005 \\
\hline & regulation_of_signal_transduction_by_p53_class_mediator_(GO:1901796) & & 0.006 \\
\hline & regulation_of_intrinsic_apoptotic_signaling_pathway_(GO:2001242) & & 0.014 \\
\hline & negative_regulation_of_apoptotic_signaling_pathway_(GO:2001234) & & 0.018 \\
\hline & regulation_of_apoptotic_signaling_pathway_(GO:2001233) & & 0.028 \\
\hline \multirow[t]{5}{*}{$12: 29562570 . .29562639$} & calcium-mediated_signaling_using_intracellular_calcium_source_(GO:0035584) & HOMER2 & 0.039 \\
\hline & regulation_of_interleukin-8_biosynthetic_process_(GO:0045414) & PRG3 & 0.039 \\
\hline & mast_cell_activation_involved_in_immune_response_(GO:0002279) & PLA2G3 & 0.039 \\
\hline & positive_regulation_of_interleukin-8_biosynthetic_process_(GO:0045416) & PRG3 & 0.039 \\
\hline & axoneme_assembly_(GO:0035082) & PLA2G3 & 0.042 \\
\hline
\end{tabular}

† GO, Gene Ontology; *a p-value, adjusted p-value

\section{Validation of the NGS Results by the qRT-PCR Analysis}

Three candidate miRNAs (hsa-miR-217, hsa-miR-143-3p, and hsa-miR-133a-5p), with the highest expression changes, were selected from the NGS data to confirm the gene expression changes. The qRT-PCR used to validate the results of NGS. The qRT-PCR findings revealed a significant decline of hsa-miR-217 expression in the achalasia tissues compared to the controls ( $p$-value $=0.004)$. These findings validated the results of the same comparison conducted by NGS. The qRT-PCR findings of hsa-miR-143-3p and hsa-miR-133a-5p, similar to NGS results, showed upregulated expression in the tissues of the patients with achalasia but, contrary to NGS, these findings were not significant ( $p$-value $=0.457$ and $p$-value $=0.840$ respectively) (Fig. 2).

\section{Discussion}


To the best of our knowledge, this study is the first study in which the miRNA expression in the tissues of the patients with achalasia was compared to the controls using the NGS approach. Our NGS data showed that 15 miRNAs had significantly differential expression in the esophageal tissues of the patients with achalasia compared to the controls. It was confirmed that miR-217 was downregulated, and miR-143-3p and hsa-miR-133a-5p were upregulated in the achalasia tissues using the stem-loop qPCR as similarly observed in the NGS results. The complete pattern of the miRNAs regulating the achalasia was obtained using the NGS approach. In a recent study using the microarray method, Shoji et al. showed that only two miRNAs (miR-361-5p and miR-130a) were upregulated in the patients with achalasia, which is contrary to the present study. This difference may be attributed to the different methods used in each study for miRNA expression analysis. Moreover, they used middle esophageal mucosa for sampling, which could potentially have different gene expression from the LES [23].

Functional annotation revealed that many miRNAs determined in our study are involved in neuronal cell apoptosis (hsa-miR-143-3p), myelination process (hsa-miR-217), and neuronal regeneration (hsa-miR-135a-5p). In accordance with our findings, Qualman et al., showed that the mechanism of esophageal dysfunction in response to neuronal destruction in the patients with Parkinson's disease is likely to be similar to that of dysphagia and achalasia [24]. Moreover, ${ }^{22}$ it was found that immune system regulation can be targeted by the hsa-miR-143-3p, which was shown to be dysregulated in our patients in the current study. Although the etiology of primary esophageal achalasia remains unknown, several hypotheses suggest that inflammation and autoimmunity are associated with its pathogenesis [25]. The histopathology analysis of the esophageal tissues, indicated lymphocytic infiltration, myenteric inflammation, and aganglionosis during the achalasia [26]. The cytotoxic autoimmune responses can potentially trigger progressive neuronal apoptosis in the achalasia tissues [27]. Evidence suggests that miRNAs play an important role in the development of neurodegenerative diseases [12].

Some of the miRNAs that significantly differentially expressed in the current study were related to cancers in other studies reports. For example, miR-217 was downregulated and acts as a tumor suppressor in several cancers such as gastric cancer [28], pancreatic ductal adenocarcinoma[29], Esophageal Squamous Cell Carcinoma (ESCC) [30], and colorectal cancer [31]. Moreover, similar to this study, reduced miR-216 expression was reported in other diseases, such as non-small cell lung cancer [32], ESCC [33], nasopharyngeal carcinoma [34], and hepatocellular carcinoma [35]. The tumor suppressor role of miR-217 and miR216 may justify the high prevalence of esophageal cancer in patients with achalasia. Despite the pathological differences between neurodegenerative diseases (such as achalasia) and cancers, new evidence suggests that they have similar regulatory mechanisms [36].

The present study indicated the upregulation of hsa-miR-143-3p in the achalasia tissues of the patients. The upregulation of miR-143 has also been shown in the $\mathrm{CD} 4^{+} \mathrm{T}$ cells highlighting the importance of this miRNA in autoimmune diseases [37]. This finding is in agreement with the role of autoimmune in the formation of achalasia.

The biological process of GO and KEGG assessments in this study demonstrated that Phosphatase and Tensin homolog (PTEN) and Sirtuin 1(SIRT1) could be significant targets of miR-217 in the achalasia (Table 4). Some studies showed that PTEN has a direct role in neurodegeneration under oxidative stress conditions [38]. Also, SIRT1 levels are associated with neurodegenerative diseases, which have a progressive and severe reduction in neuronal cells [39]. These findings could be in line with the role of neurodegeneration in the development of achalasia.

Interestingly, our findings identified some genetic factors related to the candidate miRNAs that have been reported in other studies on achalasia. For instance, the HLA genes which were showed targeted by miR-122-5p in this study were related to achalasia in the previous study [40]. In the current study, some immune modulator genes, including Interleukin 10(IL-10) and Interleukin 23 Receptor(IL-23R), were targeted by hsa-miR-143-3p and hsa-miR-216a-5 respectively $[41,42]$. Accordingly, these findings highlight the role of immunity and inflammation in the initiation and progression of achalasia (Table S2).

This research showed that miR-383-5p was downregulated in the patient with achalasia who had a poor response to the dilatation treatment. This miRNA might play a potential prognostic role for the prediction of the response to the treatment in the patients with achalasia. However, further studies are needed to confirm this finding. Other studies have introduced the hsa-miR-383 as a tumor suppressor with a decreased level in the glioma, medulloblastoma, and testicular embryonal carcinoma cells [43]. Our results demonstrated that dysregulated miR-216b could target the Tropomyosin (TPM), the gene encoding the beta-tropomyosin and playing an important role in the regulation of the calcium-dependent muscle contraction. Changes in the TPM expression have been shown in a previous study on the achalasia tissues [41]. These findings may emphasize the neuromuscular process in the pathogenesis and development of the achalasia (Table 4).

Other findings of the present study indicated that has-miR-135 was downregulated only in the patients with a good response to the treatment. Some studies have shown that the induction of miR-135a expression in different types of cancers could suppress cell proliferation through target genes (c-MYC, STAT6, SMAD5, and BMPR2), and miR-135a has also been introduced as a potential predictor of treatment outcome in some cancers [44]. This study confirmed that these target genes are significant targets of has-miR-135 in achalasia (Table 4).

This investigation found that Caveolin1 (CAV1) involving in the calcium signaling pathway could be a significant target of hsa-miR-3609 and hsa-miR-194-5p differentially expressed in the achalasia tissues of the patients. This finding is in line with a study that the CAV1target gene was differentially expressed in the achalasia tissues and was shown to have a possible function related to the achalasia pathogenesis [41]. It is generally accepted that calcium channel blockers can support LES relaxation and esophageal peristalsis in patients with achalasia [45]. This provides further support for the role of candidate miRNAs in the etiology of achalasia.

\section{Conclusion}

In conclusion, the results of the current study provide a comprehensive analysis of miRNA expression in the achalasia and may be used as a basis for future studies to investigate the role of candidate miRNAs in the etiology of achalasia. A significant downregulation was observed in the hsa-miR-217 in the LES 
samples of the patients with achalasia with significant enrichment in myelination process ontology. Furthermore, the NGS miRNA expression profiling might be a suitable platform to classify the patients with achalasia into different response groups concerning the outcome of dilatation treatment.

\section{Abbreviations}

LES: Lower Esophageal Sphincter; miRNA: microRNA; NGS: Next Generation Sequencing; KEGG: Kyoto Encyclopedia of Genes and Genomes; cDNA: DNA complementary to RNA; qRT-PCR: quantitative Reverse Transcription Polymerase Chain Reaction; rRNA, ribosomal RNA; tRNA, transfer RNA; $\triangle \triangle \mathrm{ct}$, double delta cycle threshold; GO: Gene Ontology; PTEN: Phosphatase and Tensin homolog; TPM: Tropomyosin; IL: Interleukin

\section{Declarations}

\section{Approval and Consent to Participate}

Declaration of Helsinki (1995) (as revised in Edinburgh (2000)) was followed throughout designing the study protocol and its implementation. This study was approved by the Ethics Committee of Golestan University of Medical Sciences (Ethics Code=31078693122415). Participation in this study was optional and informed written consent was obtained from all the participants and their anonymity was preserved. Test results of the participants were confidential and were only available to the physician and the moderator of the project.

\section{Consent for Publication}

Analyzed and aggregated information did not contain any personal data. So, the authors declare the "Not applicable" statement regarding Consent for Publication in this section.

\section{Availability of data and materials}

The datasets used and analyzed in the current study are available from the corresponding author on reasonable request.

\section{Competing interests}

The authors declare that they have no competing interests.

\section{Funding information}

This work was supported by the Golestan University of Medical Sciences (grant no.: 940208018). The funder has no role in study design, data collection, and analysis, decision to publish, or preparation of the manuscript.

\section{Author contributions}

A.M., J.M, SJ.M, and M.K contributed to the study conception and design. Material preparation, data collection, and analysis were performed by M.G, MR.B, M.SJ, N.J, N.F, and N.B. The first draft of the manuscript was written by M.G and all authors commented on previous versions of the manuscript. All authors read and approved the final manuscript.

\section{Acknowledgments}

The authors would like to thank all the participants who have donated their samples to the study. The authors also thank the staff of the DDRC in the Shariati Hospital affiliated with Tehran University of Medical Sciences for their collaboration.

\section{Authors' Information}

${ }^{1}$ Golestan Research Center of Gastroenterology and Hepatology, Golestan University of Medical Sciences, Gorgan, Iran. ${ }^{2}$ Autoimmune and Motility Disorders Research Center, Digestive Diseases Research Institute, Tehran University of Medical Sciences, Tehran, Iran. ${ }^{3}$ Department of Genetics, Faculty of Biological Sciences, Tarbiat Modares University, Tehran, Iran. ${ }^{4}$ Department of Animal and Poultry Science, College of Aburaihan, University of Tehran, Tehran, Iran. ${ }^{5}$ Metabolic Disorders Research Center, Golestan University of Medical Sciences, Gorgan, Iran. ${ }^{6}$ Department of Microbiology, Faculty of Medicine, Golestan University of Medical Sciences, Gorgan, Iran. ${ }^{7}$ Department of Biostatistics, Faculty of Health, Golestan University of Medical Sciences, Gorgan, Iran

\section{References}

1 Triadafilopoulos G, Patti MG, Gullo Ret al. . The Kagoshima consensus on esophageal achalasia Dis Esophagus. 2012;25:337-348.

2 Sadowski D, Ackah F, Jiang B, Svenson L. Achalasia: incidence, prevalence and survival. A population-based study Neurogastroenterol Motil. 2010;22:e256-e261.

$3 \quad$ Farrokhi F, Vaezi MF. Idiopathic (primary) achalasia Orphanet J Rare Dis. 2007;2:38.

4 Ghoshal UC, Daschakraborty SB, Singh R. Pathogenesis of achalasia cardia World journal of gastroenterology : WJG. 2012;18:3050-3057.

Ates F, Vaezi MF. The pathogenesis and management of achalasia: current status and future directions Gut and liver. 2015;9:449. 

of achalasia and implications for future treatment World J. Gastroenterol. 2016;22:7892; Park W, Vaezi MF. Etiology and pathogenesis of achalasia: the current understanding Am J Gastroenterol. 2005;100:1404-1414.

7 Bartel DP. MicroRNAs: genomics, biogenesis, mechanism, and function Cell. 2004;116:281-297.

8 Furer V, Greenberg JD, Attur M, Abramson SB, Pillinger MH. The role of microRNA in rheumatoid arthritis and other autoimmune diseases Clin. Immunol. 2010;136:1-15.

$9 \quad$ Fang Y, Fang D, Hu J. MicroRNA and its roles in esophageal cancer Med Sci Monit. 2012;18:RA22-RA30.

10 Singh RP, Massachi I, Manickavel Set al. . The role of miRNA in inflammation and autoimmunity Autoimmun. Rev. 2013;12:1160-1165;Tahamtan A, Inchley CS, Marzban Met al. . The role of microRNAs in respiratory viral infection: friend or foe? REV MED VIROL. 2016;26:389-407.

11 Wang C, Ji B, Cheng B, Chen J, Bai B. Neuroprotection of microRNA in neurological disorders (Review) Biomed Rep. 2014;2:611-619.

12 Kye MJ, Inês do Carmo GG. The role of miRNA in motor neuron disease FRONT CELL NEUROSCI. $2014 ; 8$.

13 Hasanzadeh A, Mikaeli J, Elahi E, Mehrabi N, Etemadi A, Malekzadeh R. Demographic, clinical features and treatment outcomes in 700 achalasia patients in Iran Middle East journal of digestive diseases. 2010;2:91.

14 Bolger A, Lohse M, Usadel B. Trimmomatic: a flexible trimmer for Illumina sequence data. Bioinformatics 30: btu170. 2014.

15 Friedländer MR, Mackowiak SD, Li N, Chen W, Rajewsky N. miRDeep2 accurately identifies known and hundreds of novel microRNA genes in seven animal clades Nucleic Acids Res. 2011;40:37-52.

16 Kozomara A, Griffiths-Jones S. miRBase: annotating high confidence microRNAs using deep sequencing data Nucleic Acids Res. 2013;42:D68-D73.

17 Kertesz M, lovino N, Unnerstall U, Gaul U, Segal E. The role of site accessibility in microRNA target recognition Nature Genet. 2007;39:1278.

18 Sturm M, Hackenberg M, Langenberger D, Frishman D. TargetSpy: a supervised machine learning approach for microRNA target prediction BMC bioinformatics. 2010;11:292.

19 Rehmsmeier M, Steffen P, Höchsmann M, Giegerich R. Fast and effective prediction of microRNA/target duplexes RNA. 2004;10:1507-1517.

20 Mohammadi-Yeganeh S, Paryan M, Samiee SMet al. . Development of a robust, low cost stem-loop real-time quantification PCR technique for miRNA expression analysis Mol Biol Rep. 2013;40:3665-3674.

21 Vantrappen G, Hellemans J. Treatment of achalasia and related motor disorders Gastroenterology. 1980;79:144-154.

22 Kahrilas PJ, Bredenoord A, Fox Met al. . The Chicago Classification of esophageal motility disorders, v3. 0 Neurogastroenterol Motil. 2015;27:160174.

23 Shoji H, Isomoto H, Yoshida Aet al. . MicroRNA-130a is highly expressed in the esophageal mucosa of achalasia patients Exp Ther Med. 2017;14:898-904.

24 Qualman SJ, Haupt HM, Yang P, Hamilton SR. Esophageal Lewy bodies associated with ganglion cell loss in achalasia.Similarity to Parkinson's disease. Gastroenterology. 1984;87:848-856.

25 Hirano I. Pathophysiology of achalasia and diffuse esophageal spasm GI Motility online. 2006.

26 Sodikoff JB, Lo AA, Shetuni BB, Kahrilas PJ, Yang GY, Pandolfino JE. Histopathologic patterns among achalasia subtypes Neurogastroenterol Motil. 2016;28:139-145.

27 Kahrilas PJ, Boeckxstaens G. The spectrum of achalasia: lessons from studies of pathophysiology and high-resolution manometry Gastroenterology. 2013;145:954-965.

28 Chen D-I, Zhang D-s, Lu Y-xet al. . microRNA-217 inhibits tumor progression and metastasis by downregulating EZH2 and predicts favorable prognosis in gastric cancer Oncotarget. 2015;6:10868.

29 Vychytilova-Faltejskova P, Kiss I, Klusova Set al. . MiR-21, miR-34a, miR-198 and miR-217 as diagnostic and prognostic biomarkers for chronic pancreatitis and pancreatic ductal adenocarcinoma Diagn Pathol. 2015;10:1.

30 Wang X, Li M, Wang Zet al. . Silencing of long noncoding RNA MALAT1 by miR-101 and miR-217 inhibits proliferation, migration, and invasion of esophageal squamous cell carcinoma cells J Biol Chem. 2015;290:3925-3935.

31 Wang B, Shen Z-I, Jiang K-wet al. . MicroRNA-217 functions as a prognosis predictor and inhibits colorectal cancer cell proliferation and invasion via an AEG-1 dependent mechanism BMC cancer. 2015;15:1. 
Wang R-T, Xu M, Xu C-X, Song Z-G, Jin H. Decreased Expression of miR216a Contributes to Non-Small-Cell Lung Cancer Progression Clin Cancer Res. 2014;20:4705-4716.

33 Dong S, Yin H, Dong Cet al. . Predictive Value of Plasma MicroRNA-216a/b in the Diagnosis of Esophageal Squamous Cell Carcinoma Dis Markers. 2016;2016.

34 Deng M, Tang H, Zhou Yet al. . miR-216b suppresses tumor growth and invasion by targeting KRAS in nasopharyngeal carcinoma $J$ Cell Sci. 2011;124:2997-3005.

35 Liu F, Zhou S, Deng Yet al. . MiR-216b is involved in pathogenesis and progression of hepatocellular carcinoma through HBx-miR-216b-IGF2BP2 signaling pathway Cell Death Dis. 2015;6:e1670.

36 Grasso M, Piscopo P, Confaloni A, Denti MA. Circulating miRNAs as biomarkers for neurodegenerative disorders Molecules. 2014;19:6891-6910.

37 Martínez-Ramos R, García-Lozano J, Lucena Jet al. . Differential expression pattern of microRNAs in CD4+ and CD19+ cells from asymptomatic patients with systemic lupus erythematosus Lupus. 2014;23:353-359.

38 Morris L, Veeriah S, Chan T. Genetic determinants at the interface of cancer and neurodegenerative disease Oncogene. 2010;29:3453-3464; Li P, Mao WM, Zheng ZG, Dong ZM, Ling ZQ. Down-regulation of PTEN expression modulated by dysregulated miR-21 contributes to the progression of esophageal cancer Dig Dis Sci. 2013;58:3483-3493.

39 Kim D, Nguyen MD, Dobbin MMet al. . SIRT1 deacetylase protects against neurodegeneration in models for Alzheimer's disease and amyotrophic lateral sclerosis EMBO J. 2007;26:3169-3179.

40 Ruiz-de-León A, Mendoza J, Sevilla-Mantilla Cet al. . Myenteric antiplexus antibodies and class II HLA in achalasia Dig Dis Sci. 2002;47:15-19.

41 Palmieri O, Mazza T, Merla Aet al. . Gene expression of muscular and neuronal pathways is cooperatively dysregulated in patients with idiopathic achalasia Sci. Rep. 2016;6:31549.

42 De León A, De La Serna J, Santiago Jet al. . Association between idiopathic achalasia and IL23R gene Journal of neurogastroenterology and motility. 2010;22:734-e218.

43 Lian J, Tian H, Liu Let al. . Downregulation of microRNA-383 is associated with male infertility and promotes testicular embryonal carcinoma cell proliferation by targeting IRF1 Cell Death Dis. 2010;1:e94; $\quad$ Xu D, Ma P, Gao G, Gui Y, Niu X, Jin B. MicroRNA-383 expression regulates proliferation, migration, invasion, and apoptosis in human glioma cells Tumor Biology. 2015;36:7743-7753; Xu Z, Zeng X, Tian Det al. . MicroRNA-383 inhibits anchorageindependent growth and induces cell cycle arrest of glioma cells by targeting CCND1 Biochem. Biophys. Res. Commun. 2014;453:833-838; Li KKW, Pang JCS, Lau KMet al. . MiR-383 is Downregulated in Medulloblastoma and Targets Peroxiredoxin 3 (PRDX3) Brain Pathol. 2013;23:413-425.

44 Ahmad A, Zhang W, Wu M, Tan S, Zhu T. Tumor-suppressive miRNA-135a inhibits breast cancer cell proliferation by targeting ELK1 and ELK3 oncogenes Genes \& genomics. 2018;40:243-251; $\quad$ Yamada Y, Hidaka H, Seki Net al. . Tumor-suppressive microRNA-135a inhibits cancer cell proliferation by targeting the c-MYC oncogene in renal cell carcinoma Cancer Sci. 2013;104:304-312.

\section{Figures}




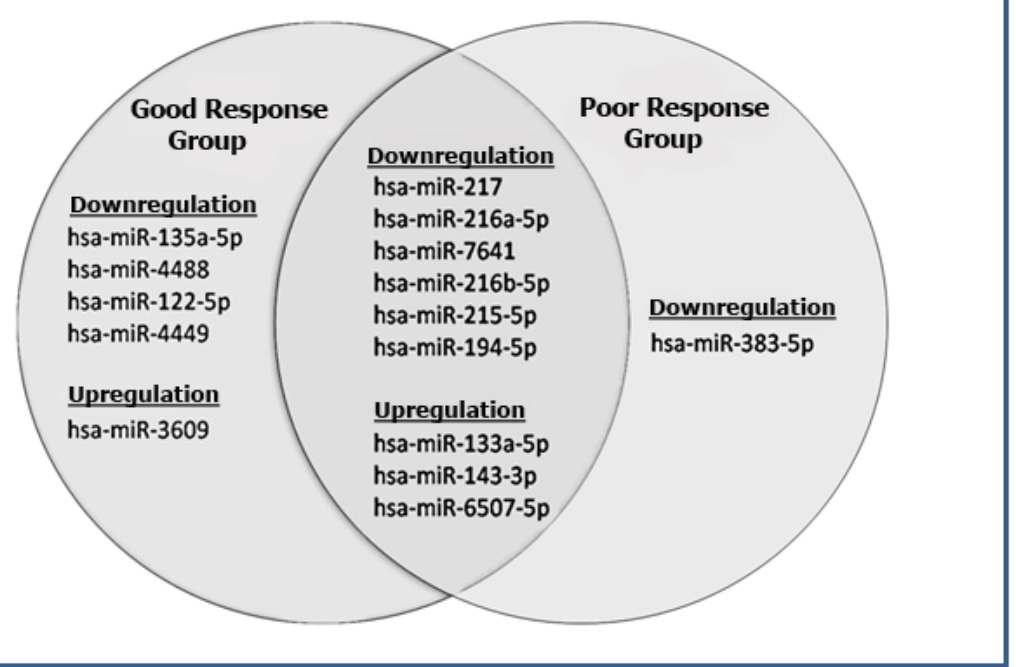

Figure 1

Candidate tissue miRNAs significantly differentially expressed in the patients with achalasia compared to the controls. Nine candidate miRNAs are common in samples of two achalasia groups. Good response group: achalasia patients with a good response to the pneumatic dilatation treatment. Poor response group: achalasia patients with poor response to the pneumatic dilation treatment.
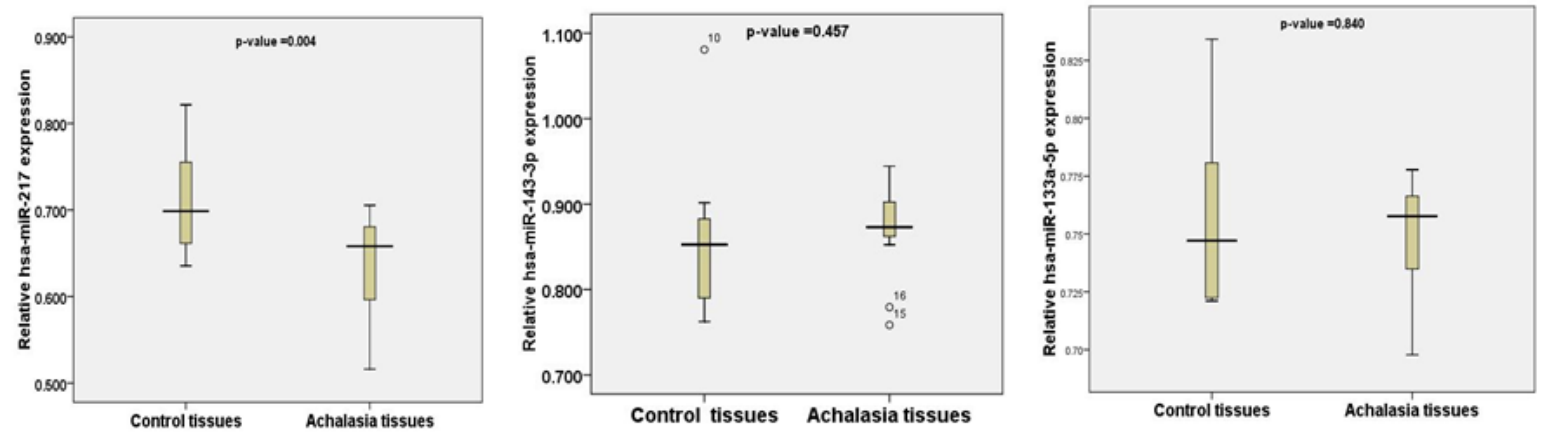

Figure 2

Relative expression of hsa-miR-217, hsa-miR-143-3p and hsa-miR-133a-5p in the esophageal tissues of the patients with achalasia compared to the controls by the quantitative Reverse Transcription Polymerase Chain Reaction (qRT-PCR) validation. Relative expression was calculated using 2-dct formula. Significant differences (p-value) have been shown in each graph.

\section{Supplementary Files}

This is a list of supplementary files associated with this preprint. Click to download.

- AdditionalfileBMCG.docx 